NOTICE: this is the author's version of a work that was accepted for publication in Neurolmage. Changes resulting from the publishing process, such as peer review, editing, corrections, structural formatting, and other quality control mechanisms may not be reflected in this document. Changes may have been made to this work since it was submitted for publication. A definitive version was subsequently published in Neurolmage, VOL.68, March 2013, http://dx.doi.org/10.1016/j.neuroimage.2012.11.048 


\section{VERTEBRAL LANDMARKS FOR THE IDENTIFICATION OF SPINAL CORD SEGMENTS IN THE MOUSE}

Megan Harrison ${ }^{1}$, Aine O’Brien ${ }^{1}$, Lucy Adams ${ }^{1}$, Gary Cowin ${ }^{2}$, Marc J. Ruitenberg ${ }^{3}$, Gulgun Sengul $^{4}$, Charles Watson ${ }^{5,6, *}$

${ }^{1}$ The University of Western Australia, Perth, WA, Australia.

${ }^{2}$ The University of Queensland, Centre for Advanced Imaging, St Lucia, Qld, Australia

${ }^{3}$ The University of Queensland, School of Biomedical Sciences, St Lucia, Qld, Australia

${ }^{4}$ Faculty of Medicine, Ege University, Izmir, Turkey.

${ }^{6}$ Neuroscience Research Australia, Sydney, NSW, Australia

${ }^{6}$ Faculty of Health Sciences, Curtin University, Perth, WA, Australia.

Email addresses: Megan Harrison 20156941@ student.uwa.edu.au, Aine O’Brien obriea05@student.uwa.edu.au, Lucy Adams li-adams@hotmail.com, Gary Cowin gary.cowin@ cai.uq.edu.au, Marc Ruitenberg m.ruitenberg@uq.edu.au, Gulgun Sengul gulgun.sengul@gmail.com, Charles Watson c.watson@ curtin.edu.au

\section{*Corresponding author}

Dr Charles Watson

Faculty of Health Sciences, Curtin University

Shenton Park Research Campus

GPO Box U1987 Perth WA 6845 Australia

Email: c.watson@curtin.edu.au; Phone: +61 892661640 


\begin{abstract}
Accurate identification of spinal cord segments in relation to vertebral landmarks is essential to surgery aimed at experimental spinal cord injury. We have analyzed a complete series of high-resolution magnetic resonance (MR) images from the mouse spine in order to delineate the boundaries of spinal cord segments in relation to vertebral landmarks. The resulting atlas can be used to plan experimental approaches that require the accurate identification of a target spinal cord segment.
\end{abstract}

\title{
Keywords
}

Spinal cord, vertebral landmarks, MR imaging, spinal cord injury, rodent 


\section{Introduction}

1.1 The mouse spinal cord has proved to be an effective model system for the study of the underlying pathophysiological mechanisms of spinal cord injury, and for testing and evaluation of the effectiveness of therapeutic interventions. It is hoped that insights from rodent animal models will lead to a better understanding of the acute and chronic morphological, cellular, and molecular consequences of spinal cord injury in humans; this should ultimately lead to better treatment and improved recovery.

Although much of the early work on spinal cord injury was conducted in rats, mice are increasingly used as a model system for spinal cord injury for a number of reasons. Firstly, mice are easy to handle and relatively inexpensive. Secondly, a large number of spinal cord genes have been precisely mapped in mice using in-situ hybridization, so that expression of candidate genes of interest can be easily assessed and mapped in different locations in the spinal cord (Allen Spinal Cord Atlas, Allen Institute for Brain Science’

http://mousespinal.brain-map.org). In the past decade, many spinal cord injury studies in mice have been combined with gene expression studies (Fan et al., 2001; Hashimoto et al., 2005; Jacob et al., 2001, Landry et al., 2006). Thirdly, a large number of genetically modified mouse strains are already available, and new strains can be generated with relative ease, thus providing a powerful genetic tool for further dissection of the pathophysiological changes in spinal cord injury at the molecular level (Basso et al, 2006).

Spinal cord injury researchers commonly report that they use vertebral spines as landmarks for experimental spinal cord injury, but rarely provide information on how the specific vertebral spines were identified, and they rarely report on which segment of spinal cord was injured. However, as the spinal cord in the adult mouse is significantly shorter than the vertebral canal (Sakla, 1969), inconsistencies in surgical approach and problems associated with the definition of anatomical landmarks during surgery can contribute to significant 
variation in the actual level of spinal cord injury. Such variation might affect the validity of those studies assessing functional recovery or the efficacy of therapeutic interventions. The lack of precise anatomic localization of the injury could be a limitation on the correct interpretation of data, particularly when independent studies from different laboratories are being compared. These observations highlight the need for a standardized way of precisely identifying spinal levels when conducting injury studies. Indeed, when we randomly surveyed a number of papers on experimental spinal cord injury in mice using PubMed (pubmed.gov). In 50 papers published over a two year period, very limited information was generally provided on the positioning of the injury and the relationship between the site of laminectomy and underlying spinal segment. These studies were based on the use of contusion $[n=24$; $48 \%$, compression $[\mathrm{n}=12 ; 24 \%]$, incomplete or complete transaction $[\mathrm{n}=13 ; 26 \%]$, and irradiation $[\mathrm{n}=1 ; 2 \%])$. Apart from one paper reporting on an injury involving the cervical region of the mouse spinal cord, the majority of these injury studies (i.e. 98\%), were related to the use of mid to lower thoracic spinal cord injury (range: T6-T11). While 48 out of 50 papers reported the vertebral level of the injury, only two studies provided information on the spinal cord segment that was injured. Only one out of these 50 papers provided information on how the target vertebra was identified.

Magnetic resonance (MR) imaging provides an ideal method for demonstrating the precise relationship between vertebral spines and bodies on the one hand and spinal cord segments on the other. Although high-resolution MR imaging techniques have been used to investigate aspects of the gross anatomy, function, and pathology of the rodent spinal cord (Bilgen et al., 2005; Falconer et al., 1994; Ford et al., 1994; Callot et al., 2007; Sandner et al., 2009, Gonzalez-Lara et al., 2009), we have not found a publication which provides a precise description of vertebral bodies relative to spinal segments across the length of the vertebral column. 
In this study, we have obtained a high resolution MR scan of the head and vertebral column of the mouse head and vertebral column in which the brain and spinal cord were left in situ. The identification of vertebral bodies and spines in these images is straightforward, and the identification of spinal cord segments has been facilitated by the recent publication of a histological atlas of mouse spinal cord (Watson et al., 2009). We have been able to use this information to identify individual spinal cord segments in the MR image stack.

In summary, this paper describes the position of each spinal cord segment in relation to the adjacent vertebral anatomy. We believe this information will enable researchers to identify the spinal cord segment targeted for injury, ultimately allowing for better standardization of surgical technique and comparisons between studies from different laboratories.

\section{Material and methods}

\subsection{Perfusion and dissection}

One C57BL/6J mouse was deeply anaesthetized using intraperitoneal injection of $0.1 \mathrm{cc}$ Lethabarb (Virbac, Australia) at a dose of $50 \mathrm{mg} / \mathrm{kg}$. Once the mouse was unresponsive to sensory stimuli, the chest cavity of the animal was opened and the animal perfused transcardially with $25 \mathrm{ml}$ of ice-cold phosphate buffered saline solution followed by $25 \mathrm{ml}$ of $10 \%$ neutral buffered formalin. After perfusion, the skin, internal organs and appendages were removed, leaving only the skull, vertebral column, and axial muscles. The specimen was postfixed for two days in $10 \%$ neutral buffered formalin, and then washed several times in phosphate buffered saline to remove all traces of formalin.

2.2 Imaging of mouse spinal cord in the spinal column 
After PBS washings, the sample was soaked in normal saline containing $0.2 \%$ Magnevist (Schering AG, Germany) for 2 days before imaging. Magnevist contains dimeglumine gadopentetate $4.69 \mathrm{~g} / 10 \mathrm{ml}$. The sample was trimmed to fit into a $15 \mathrm{~mm}$ diameter glass tube (110 mm long) while submerged in the soak solution. Samples were imaged on a Bruker (Ettlingen, Germany) AV700 magnetic resonance microimaging system consisting of a 16.4T magnet interfaced to an AVANCE II spectrometer running Paravision 4 using a $15 \mathrm{~mm}$ RF coil. The imaging protocol was a 3D gradient echo sequence with the following parameters: $\mathrm{TR}=40, \mathrm{TE}=5.2$, pulse angle $=$ Ernst angle $\left(30^{\circ}\right)$, field-of-view $=13 \times 13 \times 32 \mathrm{~mm}$, acquisition matrix $=440 \times 440 \times 512$, zero filled to give an image matrix of $512 \times 512 \times 512$ for an image resolution of $25.4 \times 25.4 \times 62.5 \mu \mathrm{m}$. The sample was moved approximately $15 \mathrm{~mm}$ between each 3D image acquisition with the number of averages between 2 and 8 and an imaging time of 5 to 18 hours per position. The sample was imaged 6 times to allow full coverage and generous overlap of the image stacks to remain within the homogenous region of the RF coil and linear region of the gradients. Total imaging time was approximately 4 days. Similar images from adjacent image stacks were selected and overlapping images removed to give a continuous stack of 1426 images. Starting at the head, each consecutive image stack was manually shifted using rotation and translation to align the anatomical features of the last image on one stack with the first image on the next stack. This minimised small shifts in the anatomical features relative to the image field-of-view due to manually moving the sample in the RF coil.

Following the completion of MR microimaging, the sample was transferred for additional imaging in a Siemens Inveon PET/CT scanner running IAW V1.4. The sample was scanned at $28 \mu \mathrm{m}$ and then down-sampled by a factor of two to give the final 3D image with an isotropic resolution of $56 \mu \mathrm{m}$. The 3D images were generated using OsiriX. 


\subsection{Identification of spinal cord segments using MR images}

The identification of transverse sections of particular spinal cord segments was based on our experience in identifying histological sections of spinal cord based on their appearance in histological stains (Nissl and acetylcholinesterase) (Watson et al., 2009). We have found that the most important landmarks for identifying segments in the limb enlargements are the motor neuron clusters. These clusters are easily seen in histological sections, and this information can be used to identify the same clusters in transverse MR sections (Figure 1). It is very difficult to decide on the segmental identity of a single isolated MR section, but examination of a series of sections allows accurate identification of most of the segments. The distinctive motor neuron clusters in each of the limb enlargements (C5 to T1 and L2 to L6) and in the upper cervical region (C1 to $\mathrm{C} 4$ ) are relatively easy to identify (Figure 1), but the motor neuron clusters in the segments from T2 to L1 lack individual characteristics in MR sections (Figure 1 - T7), and the boundaries must be allocated on a proportional basis after the T1 and L2 segments have been identified. This proportional allocation is justified by the fact that the segments from T2 to L1 are of approximately equal length (based on direct measurement of segment length in two dissected specimens in which the spinal roots were exposed).

From a practical point of view, the first step in segmenting the spinal cord is to identify the forelimb and hindlimb enlargements of the spinal cord. In the forelimb enlargement, three distinct regions can be identified in MR images - C5 and C6, C7 and C8, and T1. The ventral horn in C5 and C6 contains two major motor neuron clusters (biceps and deltoid), whereas in C7 and C8 there are five major clusters (pectoral, triceps, latissimus dorsi, forelimb flexor, and forelimb extensor muscles) (Figure 1). The T1 segment has only one major cluster - the large round group of motor neurons in the extreme dorsolateral part of the ventral horn that supplies the hand muscles. Following this separation of the forelimb enlargement into three regions, the regions containing the $\mathrm{C} 5 / \mathrm{C} 6$ and $\mathrm{C} 7 / \mathrm{C} 8$ groups can each be arbitrarily divided 
into two segments. Once the segments of the forelimb enlargement have been identified, the upper cervical region $(\mathrm{C} 1-\mathrm{C} 4)$ can be segmented. In this region, the boundaries of the four segments can initially be defined on the assumption that the segments are of similar length. The identity of each segment can be confirmed by the identification of the motor neuron clusters associated with the spinal accessory nerve (trapezius and sternocleidomastoid muscles) in C2 to C4 and the phrenic nucleus in C4 (see Watson et al., 2009 for details). The segments of the hindlimb enlargement can be distinguished on the same basis as those of the forelimb. In fact, the rostrocaudal pattern of motor neuron clusters for the hindlimb is very similar to that of the forelimb. In the case of the hindlimb, there are two rostral segments with two major motor neuron clusters (the quadriceps and adductor groups of L2 and L3), two subsequent segments with five major groups (the hamstring, gluteal, leg flexor and leg extensor groups of L4 and L5), followed by a single segment with a round dorsolateral group (the foot motor neurons of L6). Because there are no unambiguous landmarks within spinal cord segments $\mathrm{T} 2$ to $\mathrm{L} 1$, this entire region must be segmented on an arbitrary basis. Because these segments are of similar rostrocaudal length, we have found that the most practical solution is to divide this stretch of spinal cord into 13 equal segments. Because there is a slight (10\%) increase in rostrocaudal length from $\mathrm{T} 2$ to $\mathrm{T} 12$, the method of arbitrary subdivision creates a small error in relation to the position of boundaries in the lower thoracic segments, but we were able to correct for this error in our analysis by identifying each of the emerging ventral roots in the MR coronal slices.

In relation to the sacral and coccygeal segments, the border between L6 and S1 is marked by the disappearance of the foot motor neuron cluster. The boundary between S2 and S3 is marked by a rapid reduction in the diameter of the spinal cord. The region from S3 to Co3 is divided on an arbitrary basis according to length. In addition to the presence of the round dorsolateral cluster of foot motor neurons in L6, a further important landmark can be used to 
confirm the location of the L6 segment. This is the appearance of the sacral dorsal commissural nucleus. The sacral dorsal commissural nucleus is a large cluster of small neurons sitting dorsal to the central canal in the segments L6 to Co3, but absent in L5 (see inset in Figure 5). The abrupt appearance of this nucleus in rostrocaudal series of sections is an excellent anatomical landmark signifying the transition from L5 to L6.

\subsection{Matching vertebral landmarks with spinal cord segments}

To match vertebral landmarks with spinal cord segments we identified vertebral spines, vertebral bodies, and disks in a sagittal view of the MR image series, and confirmed these features with reference to the CT series (Figure 4). We recorded the number of the slices in which each feature was present. Using this data, we constructed a complete rostrocaudal diagram of the position of each vertebral landmark in the sagittal plane in a spreadsheet file. Within this file each of the skeletal structures (vertebral body, vertebral spine, vertebral disc, rib) was recorded for each slice (Figure 2). Next we examined the spinal cord in each coronal slice in a rostrocaudal series from the pyramidal decussation to the filum terminale. Using the segmentation methodology described above we established the number of slices that corresponded to each spinal cord segment. We recorded this data in the same spreadsheet file we constructed for the vertebral landmarks (Figure 2). Finally, we marked the boundaries between spinal cord segments on the spreadsheet representing the whole sagittal image series (Figures 5 to 8). We also identified each ventral root in the series of coronal spinal cord slices and recorded the slices in which the root emerged from the spinal canal (Figure 2). Figure 3 shows the ventral and dorsal roots as they emerge from the T1 spinal cord segment.

\section{Results}

3.1 The relationship between vertebral bodies, vertebral spines, and spinal cord segments 
The mouse vertebral column typically consists of 7 cervical, 13 thoracic, 6 lumbar, 4 sacral and 28 caudal vertebrae (Cook, 1965; Green, 1941). Since no anatomical boundaries mark the junction of spinal cord segments, a segment can only be defined as the region of origin of each pair of spinal nerves. Thirty-four pairs of spinal nerves ( 8 cervical, 13 thoracic, 6 lumbar, 4 sacral, and 3 coccygeal) arise from the spinal cord. There are thus 34 segments in the mouse spinal cord: 8 cervical (named $\mathrm{C} 1$ to $\mathrm{C} 8$ ), 13 thoracic (T1 to T13), 6 lumbar (L1 to L6), 4 sacral (S1 to S4) and 3 coccygeal (Co1 to Co3) (Watson and Kayalioglu, 2009). Note that there are 8 cervical cord segments, but only 7 cervical vertebrae. This is an anomaly created by the fact that spinal roots $\mathrm{C} 1$ to $\mathrm{C} 7$ are named for the vertebra that succeeds them, whereas each thoracic, lumbar, sacral, and coccygeal root is named after the vertebra that precedes it. This creates a nomenclatural gap for the root emerging between vertebrae $\mathrm{C} 7$ and $\mathrm{T} 1$, and this root is therefore called C8.

Figures 5 to 8 are MR images of midline sagittal sections of different regions of mouse vertebral column and spinal cord. Bony structures, intervertebral discs, axial muscles, ligaments, and segments of the spinal cord have been identified and labeled.

\subsection{Cervical landmarks}

Figure 5 is an annotated MR image of the cervical region and the caudal part of the head. At the most rostral part of Figure 5, the squamous occipital bone and basioccipital bone can be seen encasing the cerebellum and hindbrain. The hindbrain is continuous caudally with the beginning of the spinal cord in this figure. Spinal cord segments $\mathrm{C} 1$ through to $\mathrm{C} 7$ can be seen in this Figure. The spinal cord segments were identified in a coronal series of high-resolution images from the same specimen (see Figure 1). Note that spinal cord segments $\mathrm{C} 1$ and $\mathrm{C} 2$ are largely aligned with their respective vertebral bodies, but more caudal segments progressively 
lose their close relationship with their named vertebral bodies, so that the $\mathrm{C} 7$ spinal cord segment is level with the C6 vertebral body.

The lower part of Figure 5 shows the anterior arch of the atlas $(\mathrm{C} 1)$ and the vertebral bodies of the axis (C2) and C3 to C6. Caudal to the axis, each vertebral body is separated by an intervertebral disc. The upper part of Figure 5 illustrates the corresponding spines associated with the anterior arch of the atlas and each vertebral body. It should be noted that the tips of the spines are positioned more caudal to their corresponding bodies in this section of the vertebral column. In addition, the spinous processes of $\mathrm{C} 1$ and $\mathrm{C} 2$ are notably larger in size in comparison to the subsequent cervical spinous processes. Note that within the dorsal aspect of Figure 5 is the nuchal ligament, which extends from the occipital bone to the lower cervical and upper thoracic spinous processes.

\subsection{Lower cervical and thoracic landmarks}

Figure 6 is an annotated MR image showing the lower cervical and thoracic regions of the vertebral column. Each of the cervical spinal cord segments C5 to C8 is of the same rostrocaudal length. The thoracic spinal cord segments T1 to T11 are each about 50\% longer than the cervical segments C5 to C8. However the rostrocaudal length of each thoracic segment is the same as that of its neighbours. Figure 6 shows that the thoracic spinal cord segments become progressively more distant from their named vertebral bodies when moving from rostral to caudal, so that the T12 segment is level with the T10 vertebral body. The lower part of Figure 6 shows the vertebral bodies of C4 through to T10, each separated by an intervertebral disc. The vertebral bodies increase in rostrocaudal length from T2 to T10. The spine associated with each vertebral body is labeled. The long spinous processes of the T7, T8, and T9 vertebrae commence level with the caudal half of their parent vertebral body but extend caudally so that the tip of the spine is level with the rostral half of the body the 
succeeding vertebra. The nuchal ligament is again evident in the right hand side of Figure 6, terminating at the level of the upper thoracic spines.

\subsection{Lumbar, sacral, and coccygeal landmarks}

Figure 7 is an annotated MR image showing the lumbar, sacral, and upper coccygeal regions of the vertebral column. There is an increasing rostrocaudal disparity between the level of named spinal cord segments and similarly named vertebral bodies. An example of this is the alignment of the L3 spinal cord segment with the T12 vertebral body.

The lower part of Figure 7 shows the vertebral bodies of T11 through to L4, each separated from its adjacent neighbours by intervertebral discs. The lumbar vertebral bodies are notably larger than those of the thoracic region. The spine associated with each vertebral body is labeled. The spinous processes of L1, L2, and L3 are increasingly larger in size compared to the thoracic spinous processes and in addition they point rostrally.

Figure 8 is an annotated MR image showing the caudal end of the spinal cord and its junction with the filum terminale at the level of the L5/L6 intervertebral disc. The vertebral bodies of L4 through to L6, the intervertebral discs, and the sacrum are labelled.

\subsection{Analysis of the MR spinal cord images}

The challenge with the analysis of the MR images is the identification of the different levels in the limb enlargements. We relied here on a section-by-section comparison of the features of the ventral horn. In some MR slices, motor neuron clusters can be made out as white spots in the presumed position of a motor neuron cluster (as identified in reference Nissl sections and diagrams) (Figure 1). Where a cluster cannot be clearly identified, the presence of a motor neuron cluster must be deduced from the Nissl images and diagrams and the shape of the ventral horn. For example, in C7 and C8 the ventral horn projects laterally into the white 
matter much further than in C5 and C6 (Figure 1). In T1 the round profile of the hand motor neurons can often be identified in a favorable MR image. Similar features are found in the ventral horn of the hindlimb enlargement. For example, in L4 and L5 the ventral horn projects laterally much further than in L2 and L3, and in L6 the round profile of the foot motor neurons can often be identified (not shown). Once the segments in the hindlimb enlargements are identified, the remaining thoracic cord can be reliably segmented on a proportional basis as described above.

\section{Discussion}

A number of different methods have been used to induce experimental spinal cord injury in mice; they include complete and incomplete transection of the spinal cord, contusion and/or compression with impactor devices, clip-or balloon-mediated compression of the spinal cord, and irradiation (Ma et al, 2001; Jakeman et al, 2000; Kwon et al, 2002; Seitz et al, 2002). In many cases, the researchers assume that the same region spinal cord will be injured in a series of experiments. Experimenters commonly report the vertebral landmark selected for surgery, but do not report on which spinal cord was injured. In some cases, the researchers my not be aware that vertebral spines and bodies do not generally lie at the same level as their corresponding spinal cord segment.

The data presented here will allow researchers to accurately predict the location of spinal cord injury after an experimental procedure. We acknowledge that the images presented here are from a single mouse, which has inherent limitations. But while it would be comforting to have primary data from a number of mice, each data series involves an unreasonable cost in terms of money and time. With scanner time being very limited, we think it is unlikely that we will have another opportunity to conduct a similarly comprehensive MR imaging session. We have, however, examined variation in the relationship between vertebral landmarks and 
lumbar spinal cord segments in a parallel project (Cowin et al., 2011), and have also been able to validate some of the thoracic landmarks in MR images of the lower spine for some of our own series of experimental spinal injuries (Blomster et al., 2012). We have found the variation in vertebral and spinal cord anatomy in these regions in the C57BL mouse to be insignificant.

Although the identification of individual spinal cord segments on MR images is challenging, a systematic approach based on clear anatomical landmarks, as explained in Section 2.3, can yield satisfactory data. It first requires a good understanding of the pattern of motor neuron clusters in the two limb enlargements, as presented in our mouse spinal cord atlas (Watson et al., 2009). After the limb enlargements have been mapped, the remaining segments can largely be defined on proportional grounds. The process of identification of motor neuron clusters is difficult and tedious and requires a complete parallel set of Nissl images. We do not expect that many researchers will be able to acquire image sets that would allow motor neuron cluster identification in their own material, especially since most will not have access to a 16.4 Tesla scanner at the present time. We therefore hope that researchers will be able to rely on the work we have done in order to predict the position of spinal cord segments in relation to vertebral landmarks for their own experimental purpose.

\section{Conclusion}

In this study, we have re-examined and documented the relationship between spinal cord segments and adjacent vertebrae by linking high-resolution MRI with histological data from a recently established anatomical atlas of the mouse spinal cord (Watson et al., 2009). We have already found that the images presented in this study to be a very useful tool for identification of specific vertebrae during surgery on the mouse spine. It is therefore hoped that these results 
will serve as a reference for standardizing spinal cord injury studies in mice, aiding surgical procedures and assessment of recovery based on anatomical location of the injury.

\section{Acknowledgements}

We thank Mr Tim Butler for assistance with tissue preparation. This work was supported in part by funding from the Queensland Nuclear Magnetic Resonance Network and from an Enabling Grant from the National Health and Medical Research Council of Australia (NHMRC) to the Australian Mouse Brain MRI Mapping Consortium.

\section{References}

Allen Atlas Portal [Internet]. Seattle (WA): Allen Institute for Brain Science. (02009. Available from: http://www.brain-map.org.

Bilgen, M., Al-Hafez, B., Berman, N.E., Festoff, B.W., 2005. Magnetic resonance imaging of mouse spinal cord. Magn. Reson. Med. 54, 1226-31.

Basso, D.M., Fisher, L.C., Anderson, A.J., Jakeman. L.B., McTigue, D.M., Popovich, P.G., 2006. Basso mouse scale for locomotion detects differences in recovery after spinal cord 1njuet in five common mouse strains. J. Neurotraum. 23, 635-659

Blomster, L.V., Cowin, G.J., Kurniawan, N.D., Ruitenberg. M.J., 2012. Detection of endogenous iron deposits in the injured mouse spinal cord through high-resolution ex vivo and in vivo MRI. NMR Biomed. Jun; Epub ahead of print.

Callot, V., Duhamel, G., Cozzone, P.J., 2007. In vivo mouse spinal cord imaging using echoplanar imaging at 11.75 T. MAGMA 20, 169-73.

Cook, M.J., 1965. Anatomy of the Laboratory Mouse. Academic Press, London. 
Cowin, G.J., Butler, T.J., Kurniawan, N., Watson, C., Wallace, R.H.,; 2011. Magnetic resonance microimaging of the spinal cord in the SOD1 mouse model of amyotrophic lateral sclerosis detects motor nerve root degeneration. Neuroimage 58, 69-74.

Falconer, J.C., Narayana, P.A., Bhattacharjee, M,B., Liu, S.J., 1994. Quantitative MRI of spinal cord injury in a rat model. Magn. Reson. Med. 32, 484-91.

Fan, M., Mi, R., Yew, D.T., Chan, W.Y., 2001. Analysis of gene expression following sciatic nerve crush and spinal cord hemisection in the mouse by microarray expression profiling. Cell Mol. Neurobiol. 21, 497-508.

Ford, J.C., Hackney, D.B., Joseph, P.M., Phelan, M., Alsop, D.C., Tabor, S.L., Hand, C.M., Markowitz, R.S., Black, P., 1994. A method for in vivo high resolution MRI of rat spinal cord injury. Magn. Reson. Med. 31, 218-23.

Gonzalez-Lara, L.E., Xu, X., Hofstetrova, K., Pniak, A., Brown, A., Foster, P.J., 2009. In vivo magnetic resonance imaging of spinal cord injury in the mouse. J. Neurotraum. 26, 75362.

Green, E.L., 1941. Genetic and non-genetic factors which influence the type of the skeleton in an 1nbred strain of mice. Genetics. 26, 192-222.

Hashimoto, M., Koda, M., Ino, H., Yoshinaga, K., Murata, A.,, Yamazaki M., Kojima, K., Chiba, K., Mori, C., Moriya, H., 2005. Gene expression profiling of cathepsin D, metallothioneins- 1 and -2 , osteopontin, and tenascin- $\mathrm{C}$ in a mouse spinal cord injury model by cDNA microarray analysis. Acta Neuropathol. 109, 165-80.

Heise, C., Kayalioglu, G., 2009a. Cytoarchitecture of the spinal cord. In "Spinal Cord. A Cristopher and Dana Reeve Foundation Text and Atlas"' (C. Watson, G. Paxinos, G. Kayalioglu, Eds.), pp. 64-80. Elsevier Academic Press, San Diego.

Heise, C., Kayalioglu, G., 2009b. Spinal cord transmitter substances. In “'Spinal Cord. A Cristopher and Dana Reeve Foundation Text and Atlas" (C. Watson, G. Paxinos, G. 
Kayalioglu, Eds.), pp. 191-198. Elsevier Academic Press, San Diego.

Jacob, J.E., Pniak, A., Weaver, L.C., Brown, A., 2001. Autonomic dysreflexia in a mouse model of spinal cord injury. Neuroscience. 108, 687-93.

Jakeman, L.B., Guan, Z., Wei, P., Ponnappan, R., Dzwonczyk, R., Popovich, P.G., Stokes, B.T., 2000. Traumatic spinal injury produced by controlled contusion in the mouse. J.

Neurotraum. 17, 299-319.

Kwon, B.K., Oxland, T.R., Tetzlaff, W., 2002. Animal models used in spinal cord regeneration research. Spine. $27,1504-1510$.

Landry, E.S., Rouillard, C., Levesque, D., Guertin, P.A., 2006 Profile of immediate early gene expression in the lumbar spinal cord of low-thoracic paraplegic mice. Behav. Neurosci. $120,1384-8$.

Ma, M., Basso, D., Walters, P., Stokes, B.T., Jakem, L.B., 2001. Behavioral and histological outcomes following graded spinal contusion injury in the C57BI/6 mouse. Exp. Neurol. 169, $239-254$

Sakla, F.B., 1969. Quantitative studies on the postnatal growth of the spinal cord and the vertebral column of the albino mouse. J. Comp. Neurol. 136, 237-47.

Sandner B, Pillai DR, Heidemann RM, Schuierer G, Mueller MF, Bogdahn U, Schlachetzki F, Weidner N. In vivo high-resolution imaging of the injured rat spinal cord using a 3.0T clinical MR scanner. J. Magn. Reson. Imaging. 2009 Mar;29(3):725-30.

Seitz, A., Aglow, E., Heber-Katz, E., 2002. Recovery from spinal cord 1njury: a new transection model in the C57b1/6 mouse. J. Neurosci. Res. 67, 337-345

Sengul, G., Watson, C.. 2012. Spinal cord. In The Mouse Nervous System (C. Watson, G. Paxinos, L. Puelles Eds.). pp 423-457. Elsevier Academic Press, San Diego. Watson, C., Paxinos, G., Kayalioglu, G., Heise, C., 2009. Atlas of the mouse spinal cord. In “The Spinal Cord. A Cristopher and Dana Reeve Foundation Text and Atlas"' (C. Watson, G. 
Paxinos, G. Kayalioglu, Eds.), pp. 308-312. Elsevier Academic Press, San Diego.

Watson, C., Kayalioglu, G., 2009. The organization of the spinal cord. In " The Spinal Cord.

A Cristopher and Dana Reeve Foundation Text and Atlas', (C. Watson, G. Paxinos, G.

Kayalioglu, Eds.), pp. 1-6. Elsevier Academic Press, San Diego.

Watson, C., Sidhu, A., 2009. Toward a Spinal Cord Ontology. In "The Spinal Cord. A

Cristopher and Dana Reeve Foundation Text and Atlas"' (C. Watson, G. Paxinos, G.

Kayalioglu, Eds.), pp. 380-383. Elsevier Academic Press, San Diego. 


\section{Figure legends}

Figure 1 - Spinal cord MR and histological images

The left hand column shows MR coronal images of spinal cord segments C4, C6, C8, and T7. The right hand column shows Nissl stained histological sections of the same level, each accompanied by a diagram of the main motor neuron clusters in lamina 9 according to the atlas of Watson et al (2009). Some of the histologically defined clusters can be identified in the MR sections. In the T4 MR slice the motor neuron clusters supplying the deltoid (De9) and rhomboid (Rh9) muscles can be identified as whitish areas. In the C6 slice, the biceps and deltoid groups can be distinguished. In the C8 slice the triceps $(\operatorname{Tr} 9)$ and pectoral $(\mathrm{Pec} 9)$ muscle groups can be identified. The position of the forearm extensor group (Fex9) in C8 is indicated by a slight bulge of the dorsolateral corner of the ventral horn. In T7 the small axial (Ax9) and intercostal (ICo9) groups are represented by whitish patches.

Figure 2 - Detail from the data spreadsheet showing vertebral and spinal cord landmarks A diagram of the data contained within a small segment of a spreadsheet in which vertebral and spinal cord landmarks were recorded. The whole spreadsheet extends from the first cervical to the last coccygeal vertebral segment and covers over $900 \mathrm{MR}$ coronal slices. The short segment shown here covers slices 617 to 664 and shows the T10 vertebral body with the discs that join it to T9 and T11. The spines of T9 and T10 are shown. The diagram shows that spinal cord segments level with the T10 vertebral body are those of T12 and part of T13. The region of emergence of the T11 root from the spinal canal is seen level with the caudal end of the T10 vertebral body.

Figure 3 - MR slice through T1 spinal cord segment showing spinal nerve roots 
An image of an MR coronal slice showing the T1 spinal cord segment. The asterisk is placed lateral the the hand motor neuron group which forms a slight bulge at this level. The dorsal (dr) and ventral (vr) roots of the T1 spinal nerve are shown. In each case, the ventral root was followed to the region of emergence from the vertebral canal and recorded in the spreadsheet described in Section 2.4 (see Figure 2 which shows the site of emergence of the T11 spinal nerve root).

Figure 4 - CT and MR images of mouse axial skeleton

The upper image is a dorsal view and the middle image is a lateral view of the axial skeleton of the mouse from which spinal cord data was extracted. The lower image is a sagittal MR slice through the axial skeleton of the same mouse, in which the vertebrae and spinal cord can be seen (see labels in Figures 5-8).

Figure 5 - Annotated sagittal MR image of cervical spine An annotated image of an MR sagittal slice covering the region from the skull to the C6 vertebra.The bodies and spines of vertebrae $\mathrm{C} 1$ to $\mathrm{C} 6$ appear as dense regions, and the intervertebral discs appear as lighter regions between the vertebral bodies. The bounadries between spinal cord segments $\mathrm{C} 1$ to $\mathrm{C} 7$ are shown as dashed lines. The nuchal ligament and parts of the occipital bone are seen as dense areas. The inset CT image of the same specimen shows the bony elements present in the region.

Figure 6 - Annotated sagittal MR image of thoracic spine An annotated image of an MR sagittal slice covering the region from the $\mathrm{C} 4$ to the $\mathrm{T} 9$ vertebra.The bodies and spines of the vertebrae appear as dense regions, and the intervertebral discs appear as lighter regions between the vertebral bodies. The bounadries between spinal 
cord segments C4 to T12 are shown as dashed lines. The inset CT image of the same specimen shows the bony elements present in the region.

Figure 7 - Annotated sagittal MR image of lumbar, sacral, and coccygeal spine An annotated image of an MR sagittal slice covering the region from the T13 to the coccygeal $3(\mathrm{Co} 3)$ vertebra. The bodies and spines of the vertebrae appear as dense regions, and the intervertebral discs appear as lighter regions between the vertebral bodies. The boundaries between spinal cord segments T13 to Co3 are shown as dashed lines. The inset CT image of the same specimen (top left corner) shows the bony elements present in the region. The inset in the lower right corner compares MR coronal slices of the L6 (left) and L5 (right) spinal cord segments. The large sacral dorsal commissural nucleus (SDCom) that first appears in L6 causes the dorsal columns to be widely separated from the central canal, whereas in L5 the dorsal columns almost touch the central canal.

Figure 8 - Annotated sagittal MR image of caudal region of the spine An annotated image of an MR sagittal slice covering the region from the L4 vertebra to the coccygeal vertebrae.The bodies and spines of the vertebrae appear as dense regions, and the intervertebral discs appear as lighter regions between the vertebral bodies. The boundary between the end of the spinal cord (the third coccygeal spinal cord segment (Co3)) and the filum terminale is shown as a dashed line. The inset CT image of the same specimen shows the bony elements present in the region. 

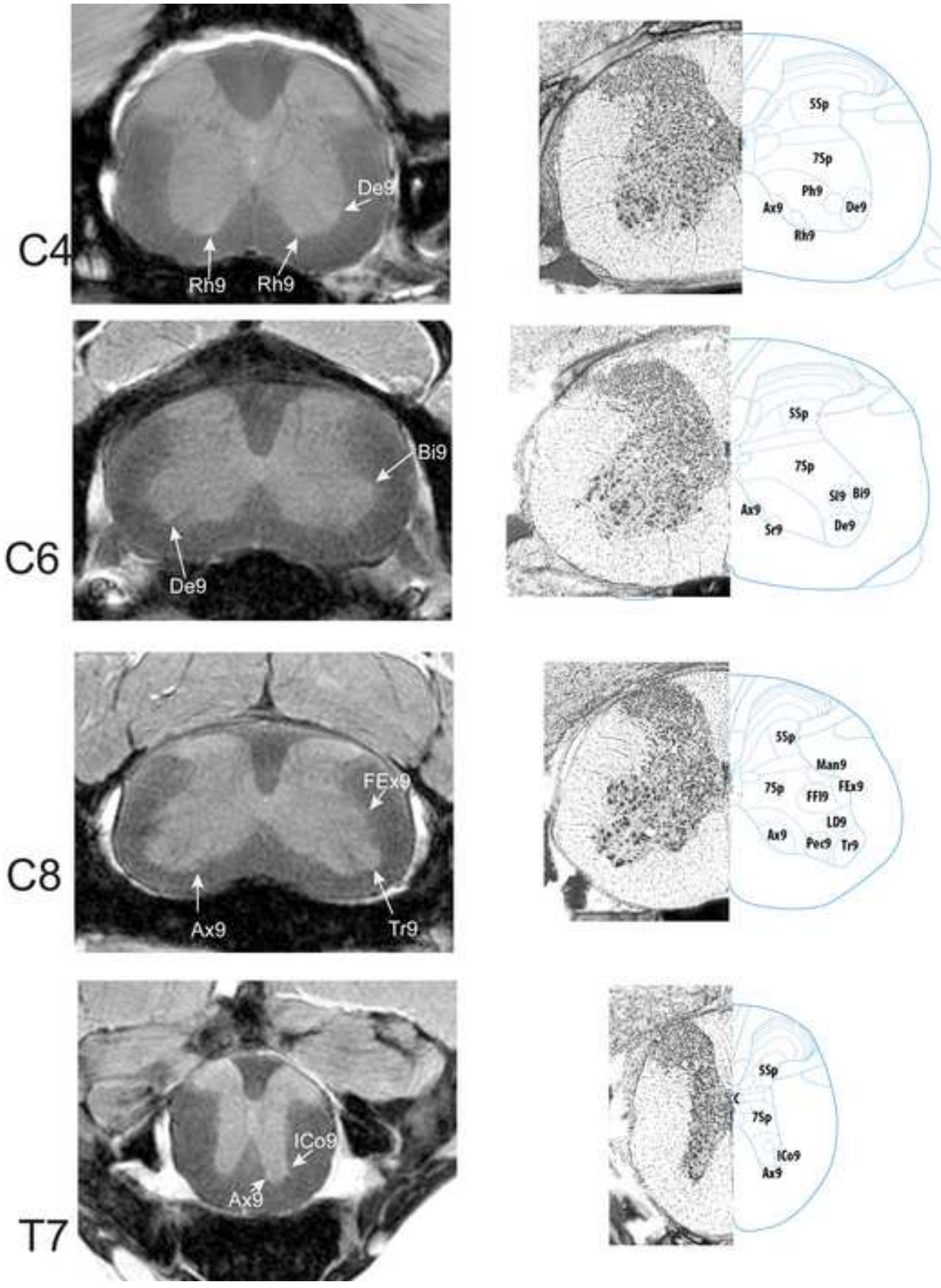

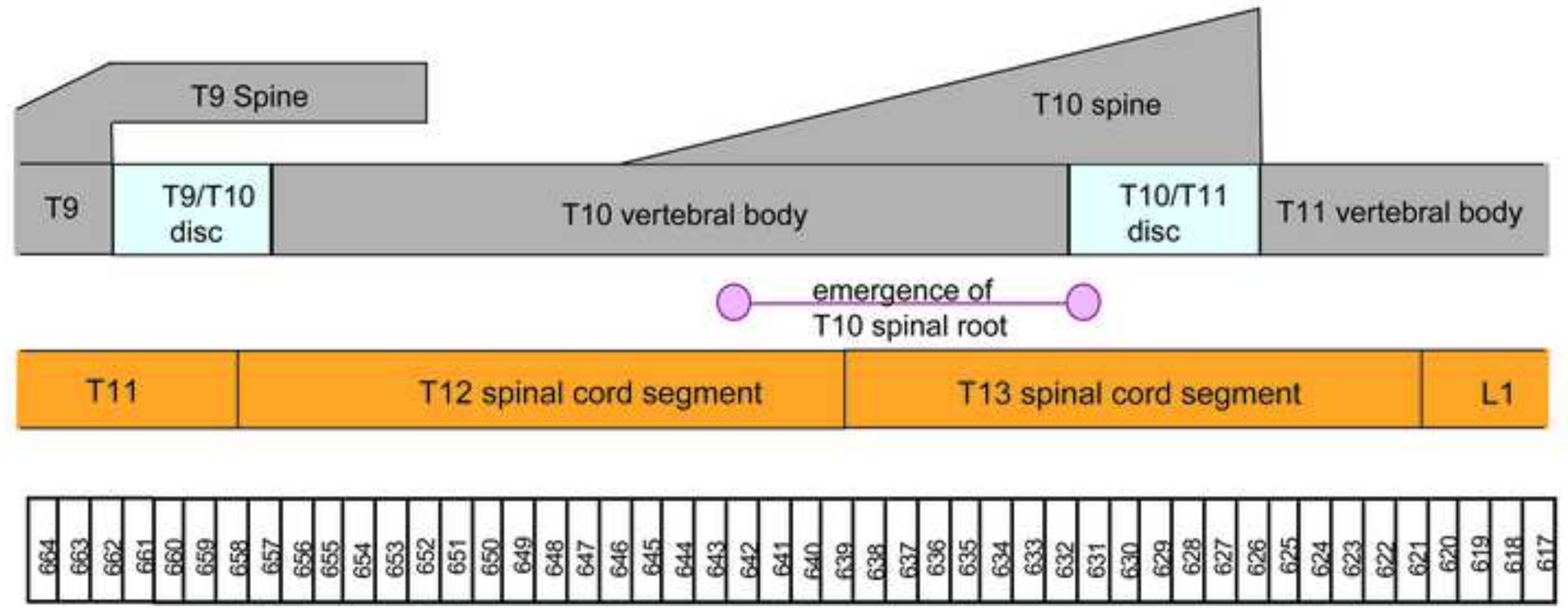
Click here to download high resolution image

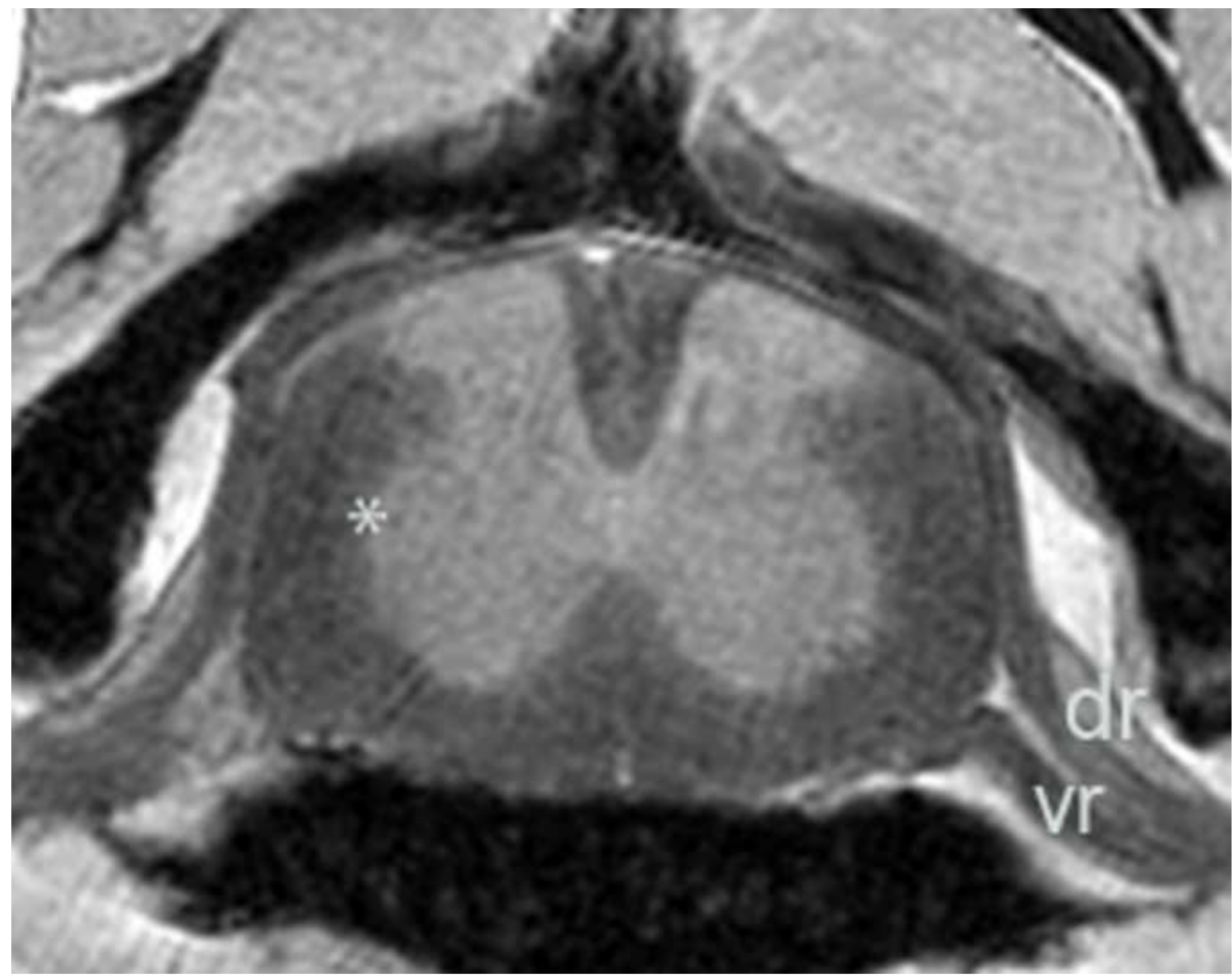



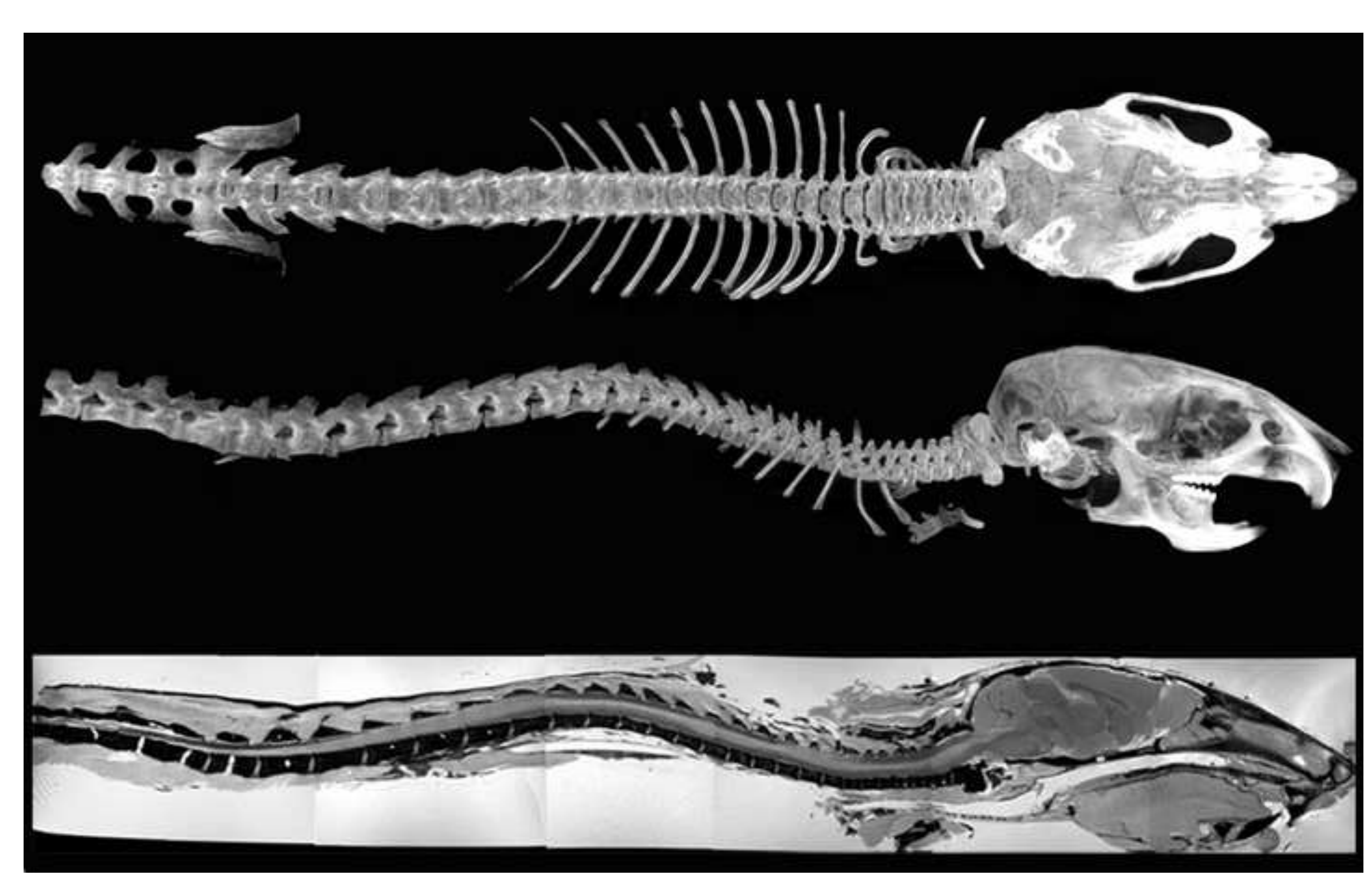

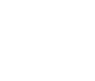

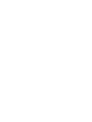
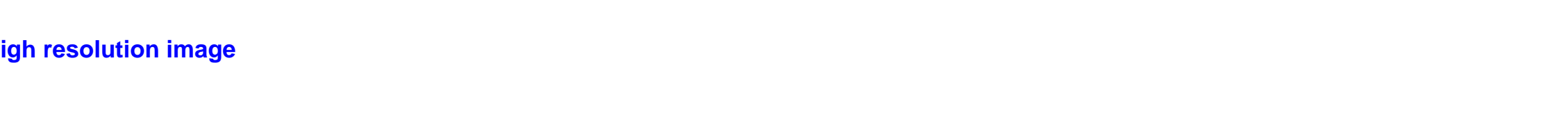

(a)

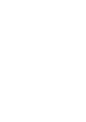

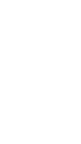

(n)

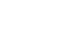




\title{
squamous occipital bone
}

\author{
cerebellum
}

C3 vertebral spine

C1

C1 vertebral spine

nuchal ligament

C2

C6 $\mathrm{C} 5 \mathrm{C} 4 \mathrm{C} 3$

C7 C6 C5 C4

C6 $\quad$ C5 $\quad$ C4

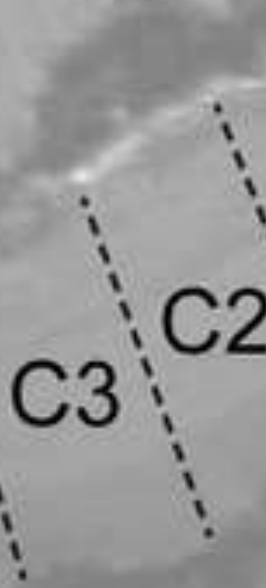

\section{basioccipital bone}

axis

atlas

$2.5 \mathrm{~mm}$

C3 vertebral body $\quad$ C2/C3 intervertebral disc 


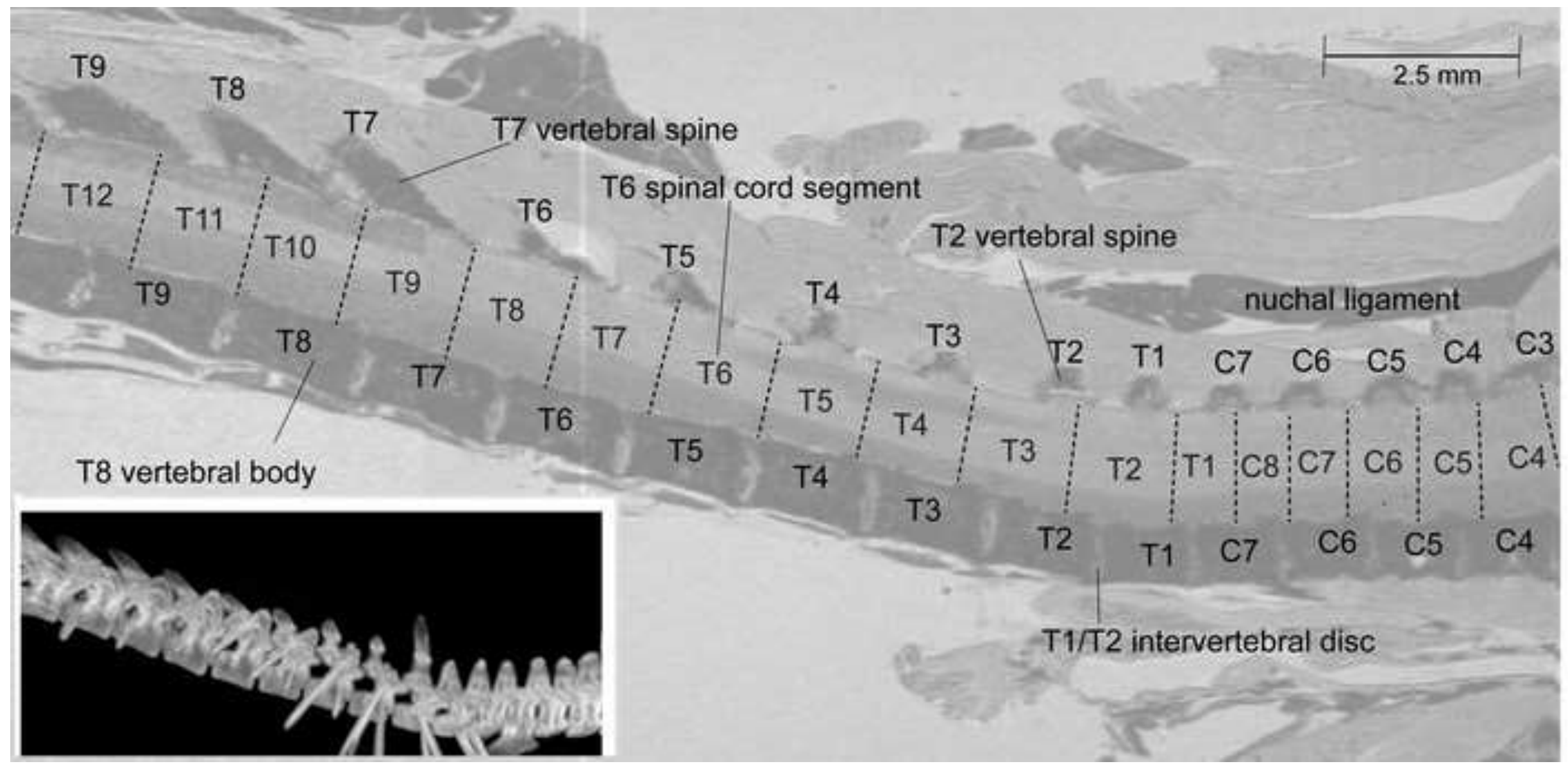



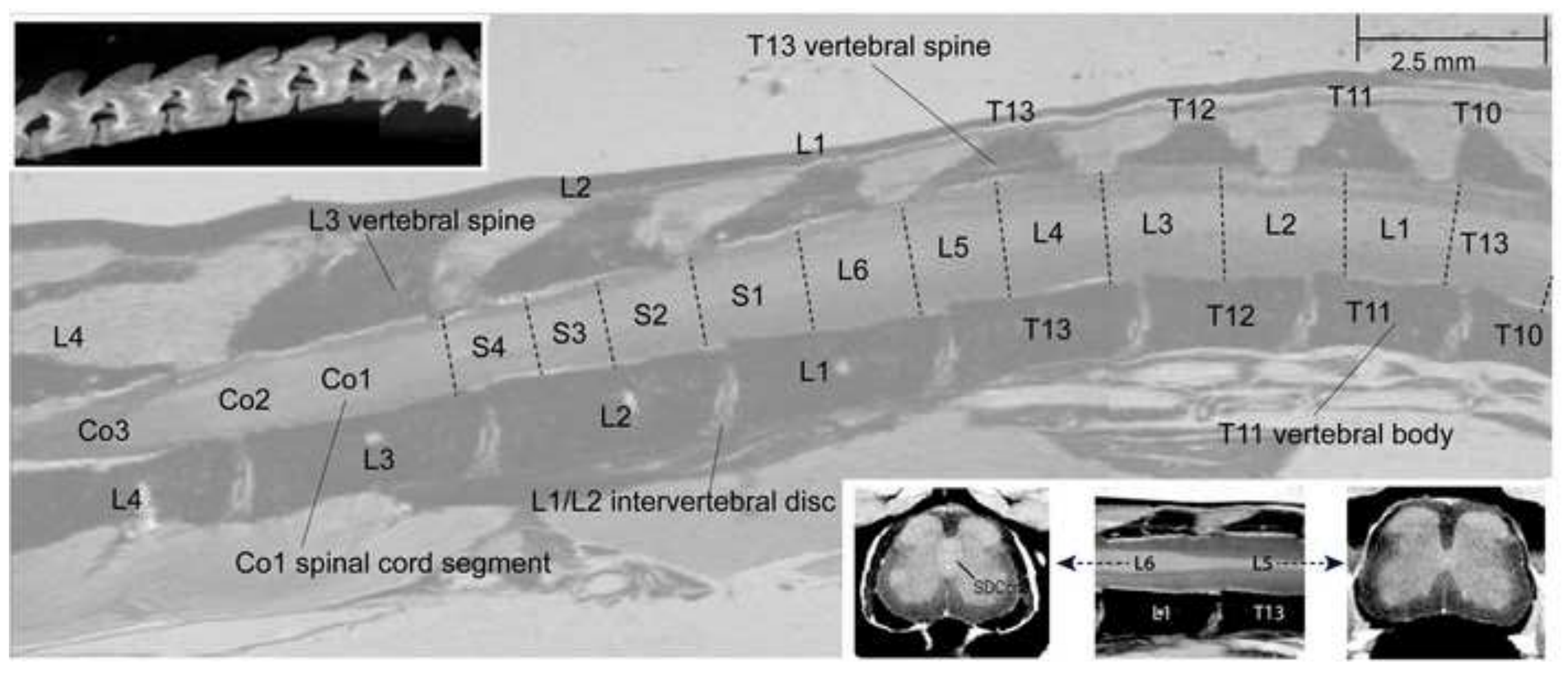

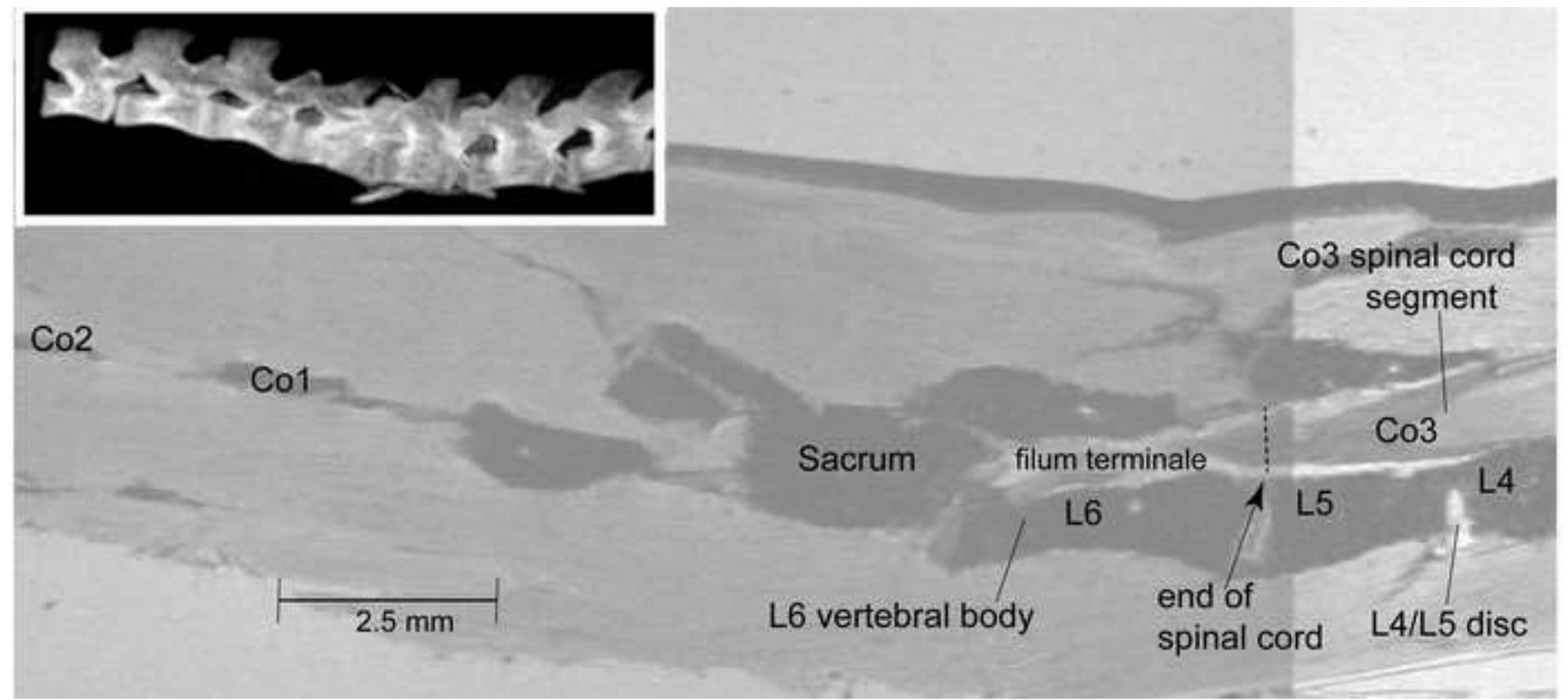